\title{
Autoimmune Hemolytic Anemia in a Patient with
} Polycythemia vera: First Description

\begin{tabular}{|l|l|l|}
\hline H.J. & Lenz \\
\hline H.D. & Waller \\
\hline
\end{tabular}

Department of Hematology/Oncology, University Hospital of Tübingen, Tübingen, FRG

\section{Key Words}

Autoimmune hemolytic anemia

Polycythemia vera

Dr. med. Heinz-Josef Lenz, Medizinische Universitätsklinik Tübingen, Abteilung II, Otfried Müller-Strasse, D-W-7400 Tübingen (FRG)

Polycythemia vera (PV) is a chronic myeloprolifer-ative disease. The pathogenesis seems to be a clonal proliferation of the hemopoietic stem cell. There are only a few case reports of autoimmune hemolytic anemia (AIHA) and myeloproliferative disorders [1] and only 2 case reports of PV and cold agglutinin disease [2, 3]. In August 1988 a 51-year-old patient was admitted with PV, which was first diagnosed in 1981. Regular phlebotomies were interrupted with a busul-fan therapy from July 1985 to January 1986 and from March to May 1988. In June 1988 an AIHA induced by warm antibody IgG with an activation of the complement system (titer of anti-C3d 1:8) was diagnosed. We started with $100 \mathrm{mg}$ prednisolone. Under steroids anemia and bilirubin normalized. The increased reti-culocytes and warm antibody IgG persisted. We had to change to $100 \mathrm{mg}$ azathioprime in November 1988 because of side effects of highdose prednisolone and persisting hemolytic parameters. Under azathioprine and low-dose prednisolone hemoglobin increased, reticulocytes fell by 56\% to $4.6 \%$, but anti-IgG titer and complement activation persisted. During the AIHA reticulocytes were between 2.4 and 56\%, LDH between 368 and 699 IU/1, hemoglobin between 7.2 and $16.6 \mathrm{~g} / \mathrm{dl}$. Because of hepatotoxicity we had to stop azathioprine after 6 months of treatment. The AIHA has now been persisting for 20 months. At the moment the patient is on low-dose prednisolone. She still suffers from hemolysis but without need for transfusion. It is the first description of AIHA induced by warm antibodies in a patient with PV. Autoimmune hemolysis because of IgG antibodies most commonly occurs in patients with lymphoreticular neoplasms. AIHA is a heterogeneous group of disor-

ders divided into three major types: paroxysmal cold hemoglobinuria, cold agglutinin disease and warm AIHA [4]. AIHA can be an uncommon adverse effect of a wide variety of drugs. Family occurrence is rare but has been reported, hypotheses are an immune-regulatory defect or a genetic defect. In our patient there was no evidence of viral infection or drug induction or a family history. A pathogenetic association between PV and AIHA has to be considered. It is our intention to increase the awareness that AIHA may complicate cases of PV. A decrease in the hemoglobin level should be investigated with that fact in mind.

References 
Arbaje YM, Beltran G: Chronic myelogenous leukemia complicated by autoimmune hemolytic anemia. Am J Med 1990;88: 197-199.

Bratusch-Marrain P, Schwarzmeier J, Kleinberger G: Akute Hämolyse bei Polycythaemia vera. Acta Med Austriaca 1979; 5:189-191.

Geissler RG, Köbberling J: Kälteagglutininkrankheit: Erst-beschreibung bei Erythroblastose und Polycythaemia vera. Klin Wochenschr 1988;66:261-263.

Shulman IA, Branch DR, Nelson JM: Autoimmune hemolytic anemia with both cold and warm autoantibodies. JAMA 1985;253:1746-1748. 